\title{
Avaliação dos trabalhos científicos de uma instituição de acordo com recomendações do comitê internacional de editores de revistas médicas
}

\author{
Evaluation of the scientific works of institution acoording to recommendations by the \\ international committe of medical magazine editors
}

Evaluación de los trabajos científicos de una institución según las recomendaciones del comité internacional de editores de revistas médicas

Ana Paula Ramos de Souza ${ }^{1 *}$, Thayná Silva Cotrim ${ }^{1}$, Karolyne Bezerra de Oliveira ${ }^{1}$, James Santos Aguiar $^{1}$, Patrícia Reis Valente ${ }^{1}$, Murilo Brandão Pimenta ${ }^{1}$, Darlinton Cardoso Fonseca ${ }^{1}$, Vinícius Francisco Fernandes Rodrigues ${ }^{1}$, Yanka Rafaela da Costa Neto Vieira ${ }^{1}$, Vitoria Silva Rodrigues ${ }^{1}$, Adriana Letícia dos Santos Gorayeb¹, Brenda Nazaré Gomes Andriolo¹.

\section{RESUMO}

Objetivo: Estimar a prevalência de trabalhos que atendem as recomendações do comitê internacional de revistas médicas. Métodos: Estudo transversal, em uma instituição do Pará, a partir da disponibilidade dos estudos apresentados nas jornadas científicas do curso de medicina, entre os anos 2015-2018. Os estudos foram avaliados quanto às recomendações propostas pelo ICMJE, classificados como adequados ou inadequados. Resultados: Foram identificados 79 projetos de trabalhos científicos entre o período de 2015 à 2018. Os resultados encontrados para o domínio "adequação ao título" somam um total de 40 estudos considerados adequados (50,6\%); no domínio "uso adequado dos descritores" $56(71,4 \%)$ trabalhos foram definidos como adequados; já para o domínio "coesão entre objetivo e conclusão" 24 (28,6\%) estudos receberam a classificação de adequado; enquanto o critério "Resumo propriamente dividido em itens", 45 $(57,1 \%)$ dos trabalhos foram classificados adequados. Conclusão: A maioria da amostra registrou de forma adequada os descritores de assunto em saúde, seguido da descrição do título atendida por um pouco mais da metade da dos estudos, enquanto a minoria seguiu a orientação para estruturação do resumo.

Palavras-chave: Educação médica, Pesquisa, Medicina na literatura.

\begin{abstract}
Objective: To estimate the prevalence of papers that meet the recommendations of the international medical journals committee. Methods: Cross-sectional study, in an institution in Pará, based on the availability of the studies presented in the scientific days of the medical course, between the years 2015-2018. The studies were evaluated for the recommendations proposed by the ICMJE, classified as appropriate or inappropriate. Results: We identified 79 scientific research projects from the period 2015 to 2018 . The results for the field "adjustment to the heading" add up to a total of 40 studies considered complete (50.6\%); in the domain "adequate use of descriptors" $56(71.4 \%)$ works were defined as adequate; for the domain "cohesion between objective and conclusion" $24(28.6 \%)$ studies were classified as adequate; while the criterion "Summary properly divided into items", $45(57.1 \%)$ of the works were classified as adequate. Conclusion: Most of the sample adequately registered the health subject descriptors, followed by the description of the title met by slightly more than half of the studies, while the minority followed the orientation for structuring the abstract.
\end{abstract}

Keywords: Education medical, Research, Medicine in literature.

${ }^{1}$ Centro Universitário Metropolitano da Amazônia (UNIFAMAZ), Belém - PA.

*E-mail: anapaula.ramoss@hotmail.com

SUBMETIDO EM: 7/2020

ACEITO EM: 8/2020

PUBLICADO EM: 10/2020 


\section{RESUMEN}

Objetivo: Estimar la prevalencia de trabajos que cumplen con las recomendaciones del comité de revistas médicas internacionales. Métodos: Estudio transversal, en una institución en Pará, basado en la disponibilidad de estudios presentados en los días científicos del curso médico, entre los años 2015-2018. Los estudios fueron evaluados de acuerdo con las recomendaciones propuestas por el ICMJE, clasificadas como adecuadas o inadecuadas. Resultados: Se identificaron 79 proyectos de trabajo científico entre el período 2015-2018. Los resultados encontrados para el dominio "adecuación al título" suman un total de 40 estudios considerados adecuados (50,6\%); en el dominio "uso adecuado de descriptores" $56(71,4 \%)$ obras se definieron como adecuadas; para el dominio "cohesión entre objetivo y conclusión", $24(28,6 \%)$ estudios se clasificaron como adecuados; mientras que el criterio "Resumen debidamente dividido en ítems", 45 (57.1\%) de las obras fueron clasificadas como adecuadas. Conclusión: La mayoría de la muestra registró adecuadamente los descriptores de temas de salud, seguidos de la descripción del título que cumplieron un poco más de la mitad de los estudios, mientras que la minoría siguió la guía para estructurar el resumen.

Palabras clave: Educación medica, Investigación, Medicina en la literatura.

\section{INTRODUÇÃO}

O baixo comprometimento com a qualidade dos textos científicos não é apenas uma grande barreira para interpretação e publicação, mas também torna difícil para profissionais entenderem o trabalho de outros pesquisadores. A deficiência na comunicação limita o impacto das pesquisas em saúde, de modo que assistentes e consumidores podem ser prejudicados. E por essa razão, os estudos precisam atender aos prérequisitos para uma boa apresentação de textos científicos (COLLIER $R, 2017$ ).

Além disso, a quantidade de artigos produzidos é crescente, tanto pelo aumento de instituições de ensino ativas em pesquisa, bem como por pressão de produtividade quantitativa nas instituições. Esse maior número de artigos faz com que seja essencial a presença de critérios para o que irá ser publicado ou não. O propósito não é o controle do que está sendo produzido, mas, sim, incentivar a originalidade e a qualidade, com a finalidade de melhor distribuir os recursos científicos (FREITAS MHA, 1999).

Algumas das formas de avaliação de produção presente no Brasil são os indicadores a exemplo do Qualis, que foi criado pela Coordenação de Aperfeiçoamento de Pessoal de Nível Superior (CAPES), e do Institute for Scientific Information (ISI), que faz parte de uma empresa norte-americana, entre outros. Porém estes apresentam restrições, tais como a presença de limitação de acervo, uma vez que não abrangem completamente as revistas existentes em especial as que são exclusivamente on-line, além de não existir entre tais indicadores um parâmetro único, o que torna mais difícil a adesão de medidas por parte dos periódicos (SANTOS LR, 2017).

Outra forma de avaliação importante ser mencionada é a iniciativa Rede EQUATOR (Enhancing the Quality and Transparency of Health Research), a qual possui em torno de 250 guidelines com a finalidade de, por meio da avaliação criteriosa de publicações científicas, evitar o desperdício de recursos humanos e financeiros em projetos de pesquisas da área da saúde. Entre esses, pode-se citar o CONSORT (Consolidation Standards of Reporting Trials) que é utilizado para ensaios clínicos randomizados e o PRISMA (Preferred Reporting Itens for Systematic Reviews and Meta-Analysis: the PRISMA Statement) para as revisões sistemáticas. O propósito desses guidelines é ofertar diretrizes práticas que avaliam a qualidade da produção, considerando a estrutura presente no artigo (título, resumo, resultados, método etc.). O uso desses guias proporciona melhorias não somente na qualidade dos artigos de dados secundários que os usam como base, mas também dos primários nos quais os autores passam a se basear neles critérios para redação dos textos (PACHECO RL, 2017).

A fim de melhorar a qualidade dos artigos para que atendam os critérios das avaliações existentes, técnicas que facilitam a qualidade da escrita científica e melhoram as ideias que compõe uma pesquisa são descritas em diversos guias como, por exemplo: as "normas para manuscritos submetidos às revistas 
biomédicas", guias para escrita científica de artigos como este costumam apresentar descrições diretas do que é necessário para uma escrita científica objetiva (CALS JW e KOTZ D, 2013; COLLIER R, 2017).

Um dos aspectos essenciais de um artigo científico é a estrutura, sendo muito utilizada a já consolidada IMRD: introdução, métodos, resultados e discussão. Essa divisão em quatro partes principais permite que os leitores possam encontrar as informações de forma mais ágil, além de tornar o texto mais objetivo e evitar redundâncias de conteúdo. Essas secções devem ter coerência entre si, com os resultados e discussão voltados a responder um objetivo científico. É importante, também, ter um título coerente e referências bem escolhidas e atualizadas (PEREIRA, 2018; GALVÃO TF, et al., 2016).

Pensando em tornar essa estruturação mais padronizada, em 1978, o Comitê Internacional de Editores de Periódicos Médicos (ICMJE) publicou um manual conhecido hoje como normas de Vancouver que uniformizaram os requisitos dos artigos científicos. Tais recomendações envolvem as seguintes secções: título, autoria, resumo, palavras-chave, introdução, método, conflito de interesse, aspectos éticos, resultados, discussão, agradecimentos, referências, tabelas e figuras. Ou seja, englobam todos os aspectos de um artigo da área da saúde. Essas orientações possuem revisões frequentes, exigindo, assim, que os pesquisadores estejam sempre se atualizando (GALVÃO TF, et al., 2016).

O objetivo para o desenvolvimento dessas recomendações da ICMJE foi revisar os padrões de boas práticas e de ética na condução da pesquisa e do relato de seus resultados, bem como de outros materiais publicados em periódicos médicos. Facilitando, assim, o processo de produção científica, acessível ao graduando de medicina. Apesar da existência essas regras, elas não são as utilizadas de forma universal. $\mathrm{Na}$ realidade, elas mais atuam como guias para instituições, revistas, bases de dados, entre outros, elaborarem suas próprias regras (DUART EF, 2013; OKKELS N, et al., 2015; BARROGA E, et al., 2019).

Entre essas orientações, no que tange a elaboração do título e do resumo de um artigo, o autor deve sempre atentar aos dados que possam direcionar melhor o leitor à pergunta científica que seu projeto pretende responder. O emprego da descrição do desenho do estudo, escrito no título é um atalho que auxilia o leitor na busca de informações pertinentes, assim como, a qualidade e objetividade das informações descritas no resumo do mesmo. Um resumo composto de dados pragmáticos que demonstrem rapidamente resultados e conclusões alcançadas tem grande importância na interpretação e qualidade da informação exposta (OLIVEIRA EFBD, et al., 2003; BRAUDAU R, et al., 2005)

Outros componentes relevantes, merecedores de destaque em virtude do processo de indexação de um artigo, são os Descritores em Ciências da Saúde (DeCS), que surgiram em 1986 pela BIREME, derivam do termo em língua inglesa Medical Subject Headings (MeSH) e são publicados pela Biblioteca Nacional de Medicina Norte Americana (U.S. National Library of Medicine), responsável pela base de dados MEDLINE, que é uma das mais completas e utilizadas na pesquisa bibliográfica na área das Ciências da Saúde (OLIVEIRA EFBD, et al., 2003; BRAUDAU R, et al., 2005; COLLIER R, 2017). As competências necessárias na graduação de medicina são os eixos saúde da família, saúde da criança, saúde da mulher, saúde do idoso.

Estes termos possuem grande valor para a indexação, pois muitos pesquisadores da área de saúde, para delimitar um campo da ciência, os utilizam na busca de informações para pesquisar sobre doenças, técnicas cirúrgicas ou mesmo escrever um trabalho. E caso eles não estejam de acordo com a nomenclatura das bases de dados, o artigo corre o risco de não ser encontrado e, portanto, não ser citado. Ademais, são importantes para a construção de revisões sistemáticas e garantem que essa seja representativa do conhecimento que existe a respeito do tópico que está sendo pesquisado (POMPEI LM, 2010).

Percebe-se, então, que a tendência é que os artigos passem por avaliações cada vez mais criteriosas, havendo a necessidade de as instituições de pesquisa exigir qualidade ao invés de quantidade aos pesquisadores. Se isso não for feito, os artigos ficarão presos a revistas de menor visibilidade e credibilidade, além de contribuir para a existência de artigos dispensáveis os quais dificultam estudos a respeito daquele tema (SANTOS LR, 2017). Por conta disso, o objetivo desta pesquisa foi estimar a prevalência de trabalhos de pesquisa que atendem as recomendações do comitê internacional de revistas médicas. 


\section{MÉTODOS}

Foi conduzido um estudo do tipo descritivo e transversal. O estudo foi realizado em uma instituição de ensino superior do Pará. Como trata-se de uma pesquisa que buscou avaliar um processo, ou seja, o foco não era o ser humano, mas sim um conjunto de documentos e os dados que são de acesso público, não houve a necessidade de avaliação por parte do Comitê de Ética em Pesquisa.

Para o recrutamento da amostra, foi realizada a solicitação para acessar os dados à Reitoria e comunicado ao comitê científico do curso de graduação em Medicina, referente aos trabalhos disponíveis para o período solicitado, caracterizando assim, amostra por conveniência. Foram incluídos todos os trabalhos científicos, encaminhados pelos alunos do curso de medicina, para as jornadas científicas que ocorreram entre janeiro/2015-setembro/2018, independente do período do aluno que submeteu o trabalho, da natureza do estudo e estágio de desenvolvimento que se encontrava.

Os dados foram coletados pelos autores do estudo, por meio de um formulário digital, padronizado, com informações sobre perfil dos seus títulos, resumos, concordância entre objetivos e conclusões, seguidos de checagem das diretivas das "Recomendações para elaboração, redação, edição e publicação de trabalhos acadêmicos em periódicos médicos" - ICMJE.

Os títulos dos trabalhos foram avaliados conforme a presença ou não da descrição do seu desenho de estudo; enquanto os resumos foram avaliados quanto a sua estruturação em itens, padrões recomendados pelo ICMJE. As palavras-chaves contidas nos trabalhos foram criteriosamente avaliadas quando a sua indexação nos Descritores em ciências da Saúde (DeCS) definidos, a partir da interpretação dos autores do projeto, como adequados ou inadequados.

A avaliação foi realizada por 2 autores de forma independente, como estratégia de prevenção de possíveis vieses de seleção. Para análise dos dados foi planejado o cálculo de frequência relativa e absoluta das variáveis de interesse. Os dados foram inseridos em uma planilha do Excel para síntese quantitativa e elaboração dos gráficos.

\section{RESULTADOS}

Foram identificados 79 projetos de trabalhos científicos entre o período de 2015 a 2018. Os resultados encontrados para o domínio "adequação ao título" somam um total de 40 estudos considerados adequados (50,6\%); no domínio "uso adequado dos descritores" 56 (71,4\%) trabalhos foram definidos como adequados; já para o domínio "coesão entre objetivo e conclusão" 24 (28,6\%) estudos receberam a classificação de adequado; enquanto o critério "Resumo propriamente dividido em itens", 45 (57,1\%) dos trabalhos foram classificados adequados (Figura 1).

Figura 1 - Gráfico de adequação segundo domínios avaliados.

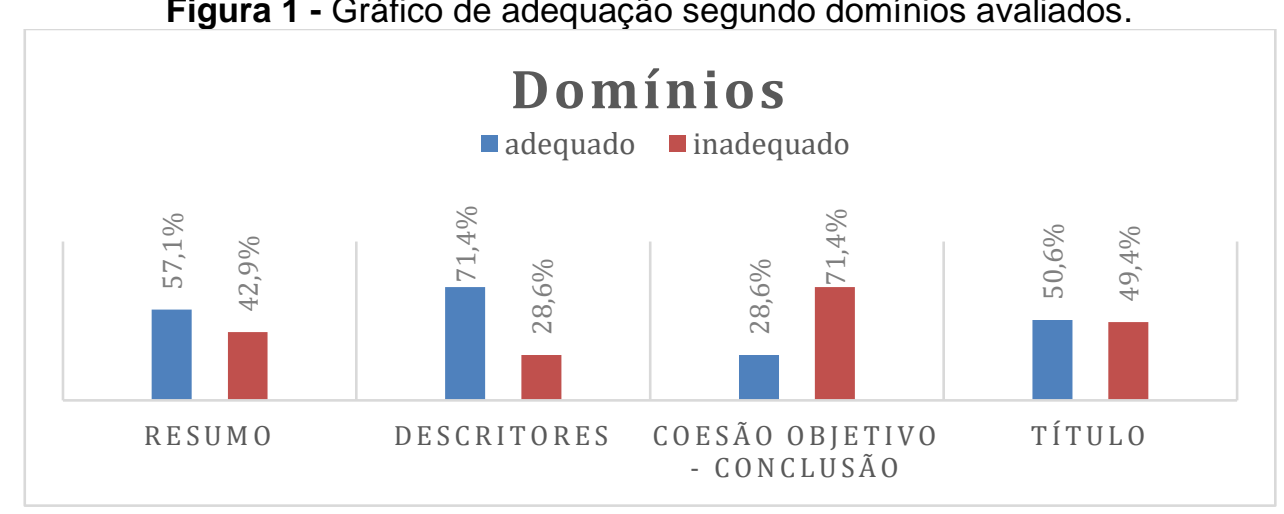

Fonte: Souza APR, et al., 2020.

Quanto aos eixos do conhecimento escolhidos pelos alunos encontrou-se o seguinte resultado: saúde do idoso com 9 trabalhos, representando 11,4\% dos projetos avaliados; saúde da família e comunidade com 32 trabalhos, correspondendo a 40.5\%; saúde da mulher com 15 trabalhos, caracterizando 19\%; saúde da criança com 2 trabalhos, representando 2,5\%; transtorno mental com 9 trabalhos, revelando 11,4\%; medicina 
alternativa com 7 trabalhos correspondendo a 8,9\%; E por fim, educação médica com 5 trabalhos, caracterizando $6,3 \%$ do total de projetos deste estudo (Figura 2).

Figura 2 - Gráfico de eixos de conhecimento.

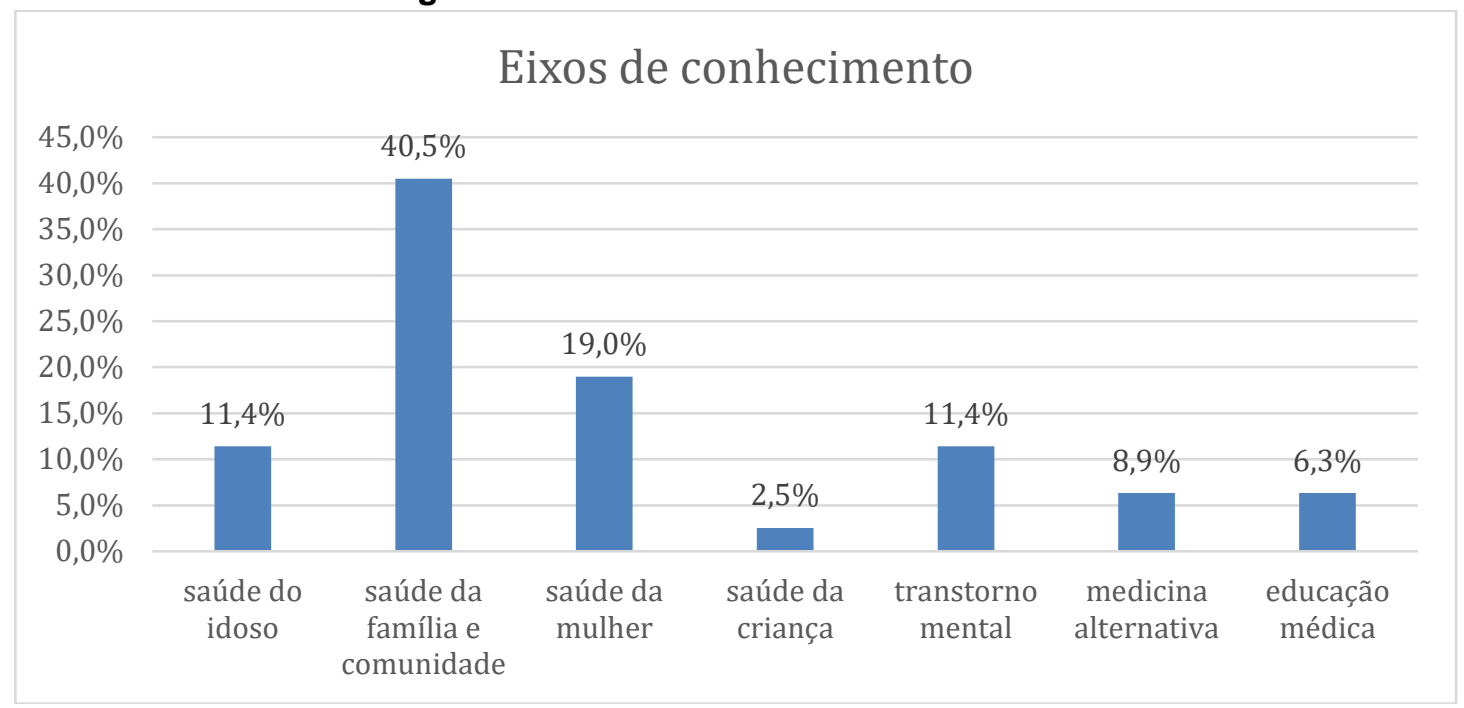

Fonte: Souza APR, et al., 2020.

\section{DISCUSSÃO}

Foi possível perceber nos resultados observados neste estudo qual a importância da iniciação científica para se alcançar uma maior adequação das boas práticas de pesquisa entre os alunos de medicina. Observase nos resultados uma alta taxa de inadequação $(71,4 \%)$ para a coesão objetivo e a conclusão, situação que demonstra baixo interesse em padronizar suas produções. Esse dado é preocupante, pois aponta a existência de possíveis problemas metodológicos nos trabalhos desenvolvidos na instituição, o que faz com que os pesquisadores não consigam obter os resultados necessários para que este responda as perguntas científicas que se propuseram no objetivo inicial. Outro fator que pode estar envolvido, citado por Shokeir (2012), diz respeito a escrever a conclusão baseada em resultados de referências anteriores ao invés de embasada nos dados novos encontrados, logo não correspondendo aos objetivos da pesquisa (OLIVEIRA EFBD, et al., 2003; CALS JW e KOTZ D, 2013; GALVÃO TF, et. al., 2016).

Pouco mais de $50 \%$ dos estudos analisados apresentaram-se com títulos considerados adequados, porém há uma quantidade significativa destes com títulos inadequados, ou seja, que não condizem com o conteúdo expresso no corpo do trabalho ou que, mais criteriosamente, não apresentam o desenho do estudo em sua composição. Isso possivelmente se deve, sobretudo pelo desconhecimento dos autores acerca das recomendações para criação de um título, o qual é indicado possuir até 40 palavras e ser um resumo rápido do que se trata o artigo para que este seja mais facilmente encontrado, precisa ser lado, específico e bem acurado em relação ao que foi abordado (OKKELS N, et al., 2015; MUBEEN SM, et al., 2017; FERNANDEZ E, et al., 2018).

A partir dos dados obtidos, verificou-se que $42,9 \%$ dos trabalhos estudados apresentam um resumo inadequado, principalmente pela ausência de estruturação em tópicos. As taxas revelam número ainda elevados, levando em consideração sua praticidade de execução e preocupante considerando sua importância.

Além de expressar de forma condensada a integralidade do artigo, o resumo tem grande relevância, pois é o primeiro contato entre o leitor e o trabalho completo. Além de que o resumo é muitas vezes a única parte da publicação que está disponível nas bases de dados, logo ele deve mostrar a relevância daquele trabalho para que os demais pesquisadores o considerem útil de ser lido integralmente, aumentando, também, a chance do artigo ser utilizado para a construção de revisões sistemáticas. Dessa forma, o autor possui a responsabilidade de detalhá-lo adequadamente, para permitir melhor interpretação do leitor e maior 
divulgação da pesquisa (ANDRADE C, 2011; CALS JW e KOTZ D, 2013; COLLIER R, 2017, HAN Z, et al., 2017; GARDNER SA, et al., 2018).

Na pesquisa em questão, $28.6 \%$ dos trabalhos analisados continham descritores não encontrados no DeCS (Descritores em Ciências da Saúde). Este critério também faz parte do contato inicial do leitor com o texto, pois os descritores são utilizados como facilitadores de busca nas bases de dados, portanto este dado revela que existe uma restrição de acesso pelos leitores à inadequação destes termos. Assim como o título, o mal uso de descritores dificulta que esses artigos sejam encontrados corretamente nas bases de dados, dificultando a disseminação daquele conhecimento. Subtende-se como principal justificativa desse resultado encontrado, o não conhecimento dos pesquisadores sobre o conceito de descritores e importância da inserção destes nas chamadas palavras-chaves (BRAUDAU R, et al., 2005; CALS JW e KOTZ D, 2013).

Dentro os assuntos pesquisados, o eixo saúde da família e comunidade foi o mais prevalente $(40,5 \%)$ concordando com a recomendação de diretrizes do curso médico, sobre a carga horária reconhecida que possa direcionar a formação profissional para o Sistema único de saúde, seguido dos eixos saúde da mulher (19\%) e saúde do idoso (11.4\%) outros de suma importância para uma maior competência médica na atenção primária. Nota-se que o graduando do curso de medicina dá importância a medicina de comunidade em seus projetos de pesquisa, remota ao mérito e relevância deste conhecimento em sua grade curricular. O sistema único de saúde tem por sua vanguarda as estratégias de saúde da família e aliada a pesquisas epidemiológicas aumentam a qualidade da assistência em saúde, incrementando a atuação do acadêmico à comunidade (BRAUDAU R, et al., 2005).

Algumas razões podem ter corroborado com a baixa adequação de alguns itens, tais como: o esforço cognitivo proveniente da escrita científica estigmatiza o processo dificultando assim a aderência dos autores ao ato de escrever e se atentar aos detalhes requeridos para a elaboração adequada de um projeto. Uma coisa que facilitaria a melhora desse processo seria um estímulo de início precoce no ambiente de pesquisa e de escrita de trabalhos científicos, de preferência ainda nos primeiros anos da vida acadêmica, com objetivo de tornar o tema mais fácil de ser compreendido, principalmente os detalhes mais específicos. Nesse sentido, a qualidade da escrita científica deve ser mais valorizada pelas instituições de ensino superior, evitando recompensas somente pela frequente produção de projetos. Infelizmente, isso não ocorre na maioria das instituições, contando ainda com entraves no ensino tradicional para tornar as matérias de escrita científica e metodologia chamativas aos mais jovens (CALS JW e KOTZ D, 2013; COLLIER R, 2017; OKKELS N, et al., 2015; SHAH J, et al., 2019; SHOKEIR AA, 2014).

Iniciativas como treinamentos práticos de como redigir um projeto, tal qual citado por aumentaria a qualidade dos textos, fazendo com que os autores colaborassem mais entre si e conseguissem resultados mais satisfatórios do que os representados neste mapeamento. É importante ressaltar que, apesar de haver o processo de triagem e editoração realizado por revistas científicas, o comprometimento dos pesquisadores com a responsabilidade com a produção científica, da concepção a escrita final, é imprescindível para a melhor qualidade dos artigos que serão publicados (MUBEEN SM, et al., 2017; GARDNER SA, et al., 2018; SHAH J, et al., 2019; GALVÃO TF, et. al., 2016).

A prática mais necessária ao escritor principiante é a escrita científica. Quanto mais um autor produz, mais sua experiência o torna apto e relevante para a ciência. $O$ hábito de escrever deve ser constantemente incentivado para apurar a técnica e consequentemente melhorar a qualidade da literatura médica (CALS JW e KOTZ D, 2013; DUARTE EF, 2013; OKKELS N, et al., 2015; COLLIER R, 2017; BARROGA E, et al., 2019).

A relevância desses dados encontra-se na necessidade observada de aumentar a aderência acadêmica ao processo de produção e pesquisa, já que, a boa escrita científica melhora a qualidade dos dados expostos e o grau de interesse do público-alvo de divulgação. Nesse contexto, a Iniciação Científica das universidades tem uma grande relevância. Tanto para o currículo da graduação, quanto pelo oferecimento de bolsas disponibilizadas pelo Conselho Nacional de Desenvolvimento Científico e Tecnológico (CNPq), por meio do Programa Institucional de Bolsas de Iniciação Científica (Pibic). Estes são considerados pontos fortes de estímulo pelos alunos. O incentivo para aumentar o número de publicações deve continuar ao longo da graduação, desde que, juntamente com o papel da universidade em estimular a escrita, haja um processo de 
estímulo as práticas guias de boas práticas científicas para assim melhorar a qualidade das mensagens a serem passadas (COLLIER R, 2017).

Algumas das limitações na execução da pesquisa foram à falta de análises relacionadas ao grau de instrução dos autores dos projetos, ou seja, categorizá-los por nível de graduação, semestre letivo, e outras variáveis possivelmente associadas aos desfechos observados, bem como o tamanho amostral e o critério de recrutamento. Outra limitação importante observada pelos autores está na inferência de que os mesmos, sendo escritores principiantes, não teriam o conhecimento do material dos editores de revistas médicas.

\section{CONCLUSÃO}

Conclui-se a partir desta pesquisa que, de modo geral, há uma baixa prevalência de estudos que seguem as práticas de escrita científica conforme recomendação dos editores de revistas médicas internacionais. A maioria da amostra registrou de forma adequada os descritores de assunto em saúde, seguido da descrição do título atendida por um pouco mais da metade dos estudos, enquanto a minoria seguiu a orientação para estruturação do resumo. Houve também baixa concordância para os domínios de coesão entre objetivo e conclusão e adequação dos títulos há uma baixa prevalência de estudos que seguem as práticas de escrita científica estimuladas pela literatura. Dessa forma, é imprescindível que haja uma conscientização mais efetiva sobre esta temática nas instituições de ensino superior, avaliando possíveis causas e aprimorando a técnica na pesquisa e publicação entre os estudantes.

\section{REFERÊNCIAS}

1. ANDRADE C. How to write a good abstract for a scientific paper or conference presentation. Indian journal of psychiatry, 2011, 53(2), 172-5.

2. BARROGA E, et al. Critical Thinking and Scientific Writing Skills of Non-Anglophone Medical Students: a Model of Training Course. Journal of Korean medical science, 2019, 34(3): e18.

3. BRAUDAU R, et al. Importância do uso correto dos descritores nos artigos científicos. Rev Bras Cir Cardiovasc 2005; $20(1)$ : 7-9.

4. COLLIER R. A call for clarity and quality in medical writing. CMAJ: Canadian Medical Association Journal, 2017, $189(46): \mathrm{e} 1407$.

5. CALS JW, KOTZ D. Effective writing and publishing scientific papers, part II: title and abstract. J Clin Epidemiol, 2013. 66(6): 585.

6. DUARTE EF. Recomendações para a elaboração, redação, edição e publicação de trabalhos acadêmicos em periódicos médicos. Epidemiol Serv Saúde. 2013 out-dez;22(4):709-32.

7. FERREIRA MJM, et al. Novas Diretrizes Curriculares Nacionais para os cursos de Medicina: oportunidades para ressignificar a formação. Interface (Botucatu), Botucatu, 2019, 23(supl. 1): e170920, 2019.

8. FERNÁNDEZ E, et al. Students' satisfaction and perceived impact on knowledge, attitudes and skills after a 2-day course in scientific writing: a prospective longitudinal study in Spain. BMJ open, 2018, 8(1), e018657.

9. FREITAS MHA. Avaliação da produção científica: considerações sobre alguns critérios. Psicol. Esc. Educ. 1998;2(3):211-228.

10. GALVÃO TF, et al. Ferramentas para melhorar a qualidade e a transparência dos relatos de pesquisa em saúde: guias de redação científica. Epidemiol. Serv. Saude. 2016;25(2):427-436.

11. GARDNER SA, et al. Supporting the Writing Productivity of Biomedical Graduate Students: An Integrated, Structured Writing Intervention. CBE life sciences education, 2018, 17(3), ar45.

12. HAN Z, et al. Engaging medical students in problem-based search and study of the biomedical literature. International journal of medical education, 2017, 8, 297-299.

13. MUBEEN SM, et al. Knowledge of scientific misconduct in publication among medical students. Educ Health, 2017, 30:140-145.

14. OLIVEIRA EFBD, et al. Avaliação de descritores na angiologia e cirurgia vascular em artigos publicados em dois periódicos nacionais. Acta Cirurgica Brasileira, 2003; 18: 01-14.

15. OKKELS N, et al, Training students in research and scientific writing. Acta Psychiatr Scand, 2015, 132: 431-432.

16. PACHECO RL. Guidelines para publicação de estudos científicos. Parte 1: Como publicar relatos e séries de casos. Diagn Tratamento. 2017;22(2):78-82.

17. PÊGO-FERNANDES PM, et al. O ensino médico além da graduação: iniciação científica. Diagn Tratamento. 2010;15(3):104-5.

18. PEREIRA MG. Dez passos para produzir artigo científico de sucesso. Epidemiol. Serv. Saude. 2017;26(3):661-664.

19. POMPEI LM. Descritores ou palavras-chave nas bases de dados de artigos científicos. FEMINA. 2010;38(5):231-232.

20. SANTOS LR, et al. Produção científica: avaliação, ferramentas e indicadores de qualidade. Ponto de Acesso. 2017;11(2):3-33.

21. SOUZA, ASR. A política de incentivo e a qualidade da publicação científica no Brasil e no Mundo. Rev. Bras. Saude Mater. Infant., Recife, v. 16, n. 1, p. 3-4, Mar. 2016

22. SHAH J, et al. Scientific writing of novice researchers: what difficulties and encouragements do they encounter? Academic medicine: journal of the Association of American Medical Colleges, 2019, 84(4), 511-6.

23. SHOKEIR AA. How to write a medical original article: Advice from an Editor. AJU. 2014;12(1):71-78. 